\title{
Jiří Vala
}

Computational modelling of thermal consumption of buildings with controlled interior temperature

In: Jan Chleboun and Pavel Kůs and Petr Přikryl and Karel Segeth and Jakub Šístek and Tomáš Vejchodský (eds.): Programs and Algorithms of Numerical Mathematics, Proceedings of Seminar. Janov nad Nisou, June 19-24, 2016. Institute of Mathematics CAS, Prague, 2017. pp. 130-143.

Persistent URL: http://dml .cz/dmlcz/703007

\section{Terms of use:}

(C) Institute of Mathematics CAS, 2017

Institute of Mathematics of the Czech Academy of Sciences provides access to digitized documents strictly for personal use. Each copy of any part of this document must contain these Terms of use.

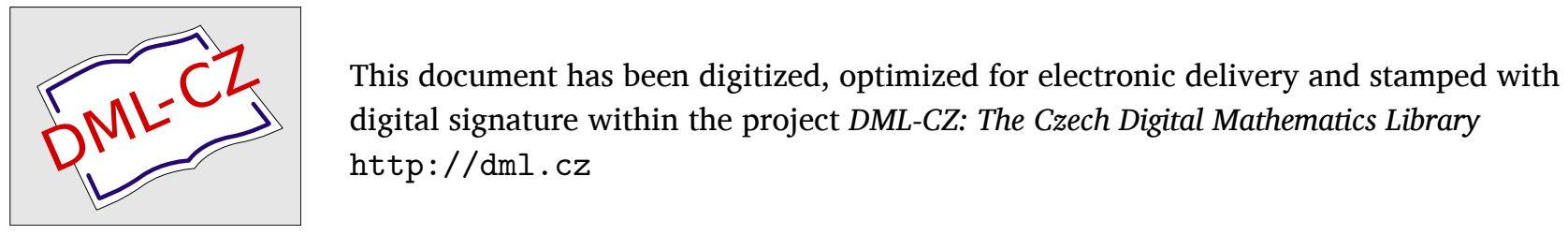


Programs and Algorithms of Numerical Mathematics 18

J. Chleboun, P. Kůs, P. Přikryl, K. Segeth, J. Šístek, T. Vejchodský (Eds.) Institute of Mathematics CAS, Prague 2017

\title{
COMPUTATIONAL MODELLING OF THERMAL CONSUMPTION OF BUILDINGS WITH CONTROLLED INTERIOR TEMPERATURE
}

\author{
Jiří Vala \\ Brno University of Technology, Faculty of Civil Engineering, \\ Institute of Mathematics and Descriptive Geometry, 60200 Brno, Veveří 95 \\ vala.j@fce.vutbr.cz
}

\begin{abstract}
New materials, structures and technologies used in civil engineering impeach traditional evaluations of the annual thermal consumption of buildings, based on the quasi-stationary estimate of the thermal resistance of the building envelope, or some operational parts of such building with the guaranteed temperature. The complete proper physical analysis, applying the principles of thermodynamics and appropriate constitutive relations for particular material layers and air in rooms, is not realistic because of the stochastic character of physical processes, of the need of identification of a lot of material characteristics, of the barely predictable behaviour of users, as well as of the limited financial budgets of investors. From the soliciting compromise solutions, this paper pays attention to the simplified formulation of dynamic behaviour of a building as a nonlinear thermal system, referring to certain analogy with the analysis of electrical circuits. Numerical solution applies the finite element technique, the method of lines and the spectral analysis of differential operators. The practical implementation is performed in MATLAB.
\end{abstract}

Keywords: building heat transfer, computational optimization, Fourier analysis

MSC: 80A20, 35K20, 65K10

\section{Introduction}

Application of advanced materials, structures and technologies in civil engineering forces new approaches to the physical, mathematical and computational analysis of both new and reconstructed buildings. Moreover, a lot of European and national directives and technical standards requires the evaluation of various thermal certificates, as the European directive [25], forcing the "passive house" properties, taken from [9], for all building actions starting 2020 and later. Unfortunately, the complete proper physical analysis of thermal behaviour of buildings, based on the application of the principles of thermodynamics and appropriate constitutive relations for particular material layers and air in rooms, is not realistic because of the stochastic character

DOI: $10.21136 /$ panm.2016.16 
of physical processes, of the need of identification of a lot of material characteristics, of the barely predictable behaviour of users, as well as of the limited financial budgets of investors. The periodically updated list www. buildingenergysof twaretools . com (formerly hosted by the US Department of Energy) contains about 500 relevant software tools; however, a physically transparent, intuitive, simple, robust and reliable numerical solver for the evaluation of energy consumption of a building, supporting the optimization of its design, too, is still not available.

The progress in this research area in the last 2 decades can be documented on [19], [15] and [22] and on numerous further references therein. Strong physical and geometrical simplifications are often overcome using some elements of soft computing by [17]: fuzzy sets, rough classifications, genetic algorithms, etc. However, some their assumptions, connecting the thermal analysis with the simplified onedimensional study of electrical circuits, relying on the finite difference and lumped mass approaches, can be removed or modified, following the weak formulation and using the properties of the Fourier decomposition. In this paper we shall come from the consideration of a building as a thermal system, suggested in [20]; for the prediction and control of energy consumption for heating of rooms (including more references) cf. [12].

\section{A model problem}

Let us consider an open set (e.g. a domain) $\Omega$ in the 3-dimensional Euclidean space $R^{3}$, supplied by the Cartesian coordinate system $x=\left(x_{1}, x_{2}, x_{3}\right)$, with its boundary $\partial \Omega$ where the local vector of (formally outward) unit normal $n(x)=$ $\left(n_{1}(x), n_{2}(x), n_{3}(x)\right)$ can be introduced. The usual notation for the Hamilton operator $\nabla=\left(\partial / \partial x_{1}, \partial / \partial x_{2}, \partial / \partial x_{3}\right)$ and for the Laplace one $\Delta=\nabla \cdot \nabla$, with the central dot used for the scalar product in $R^{3}$, will be used. Moreover, let us consider a time interval $I=[0, \tau]$ with some real positive $\tau$; the limit passage $\tau \rightarrow \infty$ is not prohibited, the dot symbol is reserved for partial derivatives with respect to the time $t \in I$, whereas the prime symbol will mean the derivative with respect to the following variable everywhere. For the brevity of notation, in this short paper we shall assume an (at least macroscopically) isotropic material (not homogeneous in general) located in $\Omega$; the straightforward generalization is left to the reader.

The conservation principle for a scalar quantity $w(x, t)$ on $\Omega$ and its flux $\eta(w(x, t))$, following [3], p. 5, and [10], p. 9, reads

$$
\dot{\varepsilon}(w)+\nabla \cdot \eta(w)=\mathcal{F}(w)
$$

where $\varepsilon(w(x, t))$ evaluates the non-stationary redistribution of $w(x, t)$ and $\mathcal{F}(w(x, t)$, $x, t)$ the sources occurring on $\Omega \times I$. Some constitutive relations for $\eta$ and $\varepsilon$ are needed: here we shall introduce them in the (rather simple) forms

$$
\begin{aligned}
& \dot{\varepsilon}(w)=\varepsilon^{\prime}(w) \dot{w}=\kappa(w) \dot{w}, \\
& \eta(w)=-\nabla \beta(w)=-\beta^{\prime}(w) \nabla w=-\lambda(w) \nabla w
\end{aligned}
$$


where $\beta$ are certain a priori known functions of $w$ (for their relation to the so-called enthalpy transform see [18], p. 252) and $\kappa$ and $\lambda$ are the material characteristics, in general functions of $w$ again (but not of $x$ and $t$ explicitly), used in most engineering applications. We shall work with the unified boundary condition

$$
\nabla \eta(w) \cdot n=\mathcal{G}(w)
$$

on $\partial \Omega$, containing the sources $\mathcal{G}(w(x, t), x, t)$; some special forms of both $\mathcal{F}$ and $\mathcal{G}$ will be introduced later. Let us remark that such definitions of $\mathcal{F}$ and $\mathcal{G}$ are open to handle relations between different quantities $w$, as temperature, velocity components or density, considered as reference variables in conservation principles for energy, momentum and mass.

Inserting only the 1st equation from (3) into (1), we obtain

$$
\dot{\varepsilon}(w)-\Delta \beta(w)=\mathcal{F}(w) .
$$

Applying the Kirchhoff transform, $\varepsilon(w)$ in (5) can be simplified to $w$, using certain modified variable $w$; for more details see [23], with several important references to [18]. In particular, for the constant values of $\kappa$ and $\lambda$ from (2) and (3) such transform is very easy. Unfortunately, in most other cases this makes namely (4) rather complicated and is not friendly to numerical approximations, thus we shall work with the original forms of (1), (2), (3) and (4) here. To solve $w$ from such system of equation of evolution, the knowledge of initial values $w_{0}(x)$ of $w(x, t)$ with $t=0$ for any $x \in \Omega$ is also needed, i.e.

$$
w(., 0)=w_{0} .
$$

Using the Green - Ostrogradskii theorem formally, we are able to convert (at least in the sense of distributions) (1), (2), (3) and (4) to

$$
(v, \kappa(.) \dot{w})+(\nabla v, \lambda(.) \nabla w)=(v, \mathcal{F}(.))+\langle v, \mathcal{G}(.)\rangle
$$

with any test function $v(x)$ from an appropriate function space introduced on $\Omega$, scalar products $(.,$.$) on L^{2}(\Omega)$ and $L^{2}(\Omega)^{3}$, or their generalizations in the sense of dualities in non-unitary spaces, similarly $\langle.,$.$\rangle on L^{2}(\partial \Omega)$, etc. Then $(7)$ can be understood as a weak (but physically transparent) condition for the conservation of a scalar quantity $w$.

A generalization of this approach to more complicated configurations, including a finite number of open sets $\Omega$ and a set of their mutual boundary parts, is straightforward, although not quite easy. We shall discuss this approach for the special case of the simplified evaluation of thermal transfer in buildings. In this case we are allowed to formulate only one principle of conservation of (thermal) energy (or enthalpy), with the (absolute) temperature $w$ as the reference variable. A building is considered as a thermal system where all particular elements and subsystems (represented by $\Omega$ here) are coupled using the interface thermal fluxes from all admissible 
adjacent elements and subsystems, as well as from the external environment. All terms (.) here, for the simplicity, refer to the possible dependence of related quantities on $w$; the significance of such dependence may be very different in practical engineering applications. In such reasonable model, $\mathcal{F}$ and $\mathcal{G}$, needed in $(7)$, come:

i) $\mathcal{F}$ from the heat sources in rooms, controlled to satisfy requirements of technical standards (which can be quantified as an optimization problem or using some results from the control theory), together with certain well-being of inhabitants (whose quantification relies to expert decisions, soft-computing or advanced statistical approaches - cf. [14]) and ([7]),

ii) $\mathcal{F}$ from the obligatory air exchange in rooms, with similar limitations as i),

iii) $\mathcal{G}$ from the heat convection, driven by external temperature development in day and year quasi-cycles, expressible as

$$
\mathcal{G}(w)=\alpha(.)\left(w_{\times}-w\right)
$$

on $\partial \Omega$ where $\alpha$ (a function of $w$ and $w_{\times}$in general) is the transfer factor and $x$ refers to the external environment, which can be repeated literally for the transfer between rooms and separating structures, too,

iv) $\mathcal{G}$ from the heat radiation by the Stefan - Boltzmann law,

$$
\mathcal{G}(w)=\sigma(.)\left(w_{\times}^{4}-w^{4}\right)=\sigma(.)\left(w_{\times}-w\right)\left(w_{\times}+w\right)\left(w_{\times}^{2}+w^{2}\right)
$$

on $\partial \Omega$ where $\sigma$ replaces $\alpha$ from iii) (its value is related usually to the theoretical one, derived for the unshaded perfectly black surface, utilizing some multiplicative correction factors),

v) $\mathcal{G}$ from the direct and diffusive solar heat radiation, due to the geographical location, slope and orientation of building surfaces, their (natural or artificial) shading, etc. - for more details see [12].

\section{Fourier approximations}

In general, using the Einstein convention for all sum indices $i, j, k \in\{1,2, \ldots, n\}$, with $n \rightarrow \infty$ theoretically, we can seek for the evolution of $w$, utilizing the multiplicative Fourier decompositions, by [1], p. 215, and [6], p. 346, in the form

$$
w(x, t)=\varphi_{i}(x) u_{i}(t)
$$

where $\varphi_{i}$ form a sufficiently rich system of functions defined on $\Omega$ (or on a union of a finite number of such open sets, as sketched above) approximating the needed space of all $\varphi$ (e.g. the Sobolev space $W^{1,2}(\Omega)$ or some weighted space derived from it - see [18], p. 14) and $u_{i}$ represent some (a priori unknown) functions of time. The choice of $\varphi_{i}$ refers frequently to various finite element techniques - cf. [5], pp. 299, 323. 
Assuming that some system of (fundamental) solutions of (7), i. e. those independent of (6)) $U_{i k}$, is available, we can rewrite (10) as

$$
w(x, t)=\varphi_{i}(x) U_{i k}(t) c_{k}
$$

with some unknown real constants $c_{k}$, satisfying (6) (at least) in the least squares (minimum variation) sense - cf. [4], p. 133. All test function in (7) can be then selected as $v=\varphi_{j}$. Consequently we have a system of linear algebraic equations

$$
\left[\left(\varphi_{j}, \kappa(.) \varphi_{i}\right) \dot{U}_{i k}+\left(\nabla \varphi_{j}, \lambda(.) \nabla \varphi_{i}\right) U_{i k}\right] c_{k}=\left(\varphi_{j}, \mathcal{F}(.)\right)+\left\langle\varphi_{j}, \mathcal{G}(.)\right\rangle .
$$

Let us notice that for practical computations the following heuristic linearization is useful: all values referenced by (.) should be estimated from the preceding time, as the initial choice for the algorithm of their successive improvement inside any time step. The proper mathematical and numerical analysis of relevant algorithms is needed; however, in this paper we shall sketch it only in one special case.

\section{Thermal performance of buildings}

For the computational analysis of thermal performance of buildings, applying the system approach, as introduced above, taking (11) and (12) to account, we can convert (7) to the matrix form

$$
M \dot{u}+K u=f+\dot{g}
$$

where the real square matrices $M$ and $K$ come for two left-side additive term of (7), together with all appropriate parts of (8) by iii) and (9) by iv), the vector $f$ is then generated by i), v) and the remaining terms from iii) and iv) and the vector $g$ by ii); another supplement to iii) is allowed to come for the properly controlled artificial heating, to save the temperature in rooms at the level required by technical standards. Roughly speaking: $M$ represents the thermal accumulation properties, $K$ the thermal insulation ones, whereas $f(t)$ and $g(t)$ (namely their additive components) correspond to various types of time-variable heat sources. The best design of a vector of unknowns $u(t)$ seems to be that it refers to temperature values on a sufficiently rich set of points characterizing the building (typically for the application of Lagrange interpolation in the finite element method); its $n$ components can be obtained from (10) in the form

$$
\left(\varphi_{i}, \varphi_{k}\right) u_{i}(t)=\left(w(., t), \varphi_{k}\right) .
$$

Let us notice the close relation of (13) to the generalized eigenvalue problem

$$
M V=V \Lambda
$$

where $\Lambda$ is a diagonal matrix of all eigenvalues and $V$ contain all eigenvectors in its corresponding columns; both $V$ and $\Lambda$ can be positive and real, which depends 
on the suitable (classical Fourier, finite element, wavelet, etc.) choice of the basis $\varphi_{i}(i \in\{1,2, \ldots n\})$. Consequently the method of variation of constants, coming from (15), in account with (6), gives the result

$$
u(t)=V \exp (-\Lambda t) V^{T} M u_{0}+V \int_{0}^{t} \exp (\Lambda(\tau-t)) V^{T}(f(\tau)+\dot{g}(\tau)) \mathrm{d} \tau .
$$

In particular, for a finite positive time step $h$, to evaluate $u^{1} \approx u(h)$ from $u^{0}$ derived from (14) and (6) (and similarly $u^{2} \approx u(2 h)$ from $u^{1}$, etc.), assuming that $f$ and $g$ are (for simplicity) linear splines defined on the set $\{0, h, 2 h \ldots\}$ with the accurate values $f^{s}=f(s h)$ and $g^{s}=g(s h)$ for $s \in\{0,1,2, \ldots\}$, we come to the result, useful for the design of practical algorithms,

$$
\begin{gathered}
u^{1}-V \exp (-\Lambda h) V^{T} M u^{0}=V(I-\exp (-\Lambda h)) . \\
\cdot\left(\Lambda^{-1} V^{T} \frac{g^{1}-g^{0}}{h}-\Lambda^{-2} V^{T} \frac{f^{1}-f^{0}}{h}\right)+V \Lambda^{-1} V^{T} f^{1}-V \Lambda^{-1} \exp (-\Lambda h) V^{T} f^{0} .
\end{gathered}
$$

The Taylor expansion $I-\exp (-\Lambda h) \approx \Lambda h$, with the error $\mathcal{O}\left(h^{2}\right)$, applied to (17), leads to the Euler explicit scheme (unstable for real buildings and climatic records frequently), whereas the implicit one can be forced by the backward integration with the exchanged role of $u^{0}$ and $u^{1}$; for the comparison to the Crank-Nicholson, etc. schemes and the detailed stability and accuracy analysis see [2], pp. 639,646. Here we intend to demonstrate namely the implementation of the influence of solar radiation to the computational algorithm based on (17) and the possibility of optimal control of artificial heating.

\section{Solar radiation}

Solar radiation can be handled using the surface thermal fluxes $\mathcal{G}$, contributing (in the relevant finite element or similar discretization - for more details see [23]) to $f$ in (13), whose evaluation as the sum of beam (direct) and diffusive radiation intensities $q_{b}$ and $q_{d}$ comes from the analysis of mutual Sun - Earth positions and from the location and orientation of a building. The preliminaries for such calculations are explained in [21], [16] and [11]; their assessment to a real building object (due to its location, orientation, surface properties, etc.) relies on [15], the detailed analysis of this type is contained in [13], p. 47.

Following [15], let us introduce the surface reflexivity $\rho$, together with certain shading factors $\rho_{b}$ and $\rho_{d}$. Let us also consider the geographical latitude $\phi$, the azimuth angle $\gamma$ (depending on the building orientation, related to south), and the angle $\beta$ between the building surface and the horizontal plane. The evaluation day and year cycles of Sun - Earth positions requires the knowledge of declination $\delta(N)=0.1302 \dot{7} \pi \sin (2 \pi(284+N) / 365)$ (presented here only for the standard year length of 365 days for simplicity) and of the day time angle $\omega(\xi)=\pi \xi$ for $\xi=\left(1-\left(t-t_{\times}\right)\right) / t_{*} ; N \in\{1, \ldots, 365\}$ refers to the day in the year, $t_{\times}$to the 
initial day time and $t_{*}$ (formally) to the standard day length. Further astronomical corrections and generalizations of $\delta(N), \omega(\xi)$, etc., are available, but not presented and implemented here, because of i) the substantial extension of computational formulae, ii) the lack of methods and input data for reasonable setting of corresponding physical characteristics. Neglecting such corrections and generalizations, the zenith angle $\varsigma$ comes from the relation

$$
\cos \varsigma=\sin \phi \sin \delta(N)+\cos \phi \cos \delta(N) \cos \omega(\xi),
$$

whereas the real angle of incidence of sunrays $\vartheta$ needs to calculate

$$
\begin{aligned}
\cos \vartheta=\cos \varsigma & +\sin \phi(\sin \delta(N)(\cos \beta-1)+\cos \delta(N) \sin \beta \cos \gamma \cos \omega(\xi)) \\
& +\cos \phi(\cos \delta(N)(\cos \beta-1) \cos \omega(\xi)-\sin \delta(N) \sin \beta \cos \gamma) \\
& +\cos \delta(N) \sin \beta \sin \gamma \sin \omega(\xi) .
\end{aligned}
$$

Making use of (18) and (19), together with the measurement results for $q_{b}$ and $q_{d}$, we are finally able to evaluate

$$
\begin{aligned}
F L U \mathcal{G}= & \rho_{b} q_{b} \min (\cos \vartheta, 0) / \min (\cos \varsigma, 0.087)+\rho_{d} q_{d}(1+\cos \vartheta) / 2 \\
& +\rho\left(q_{b}+q_{d}\right)(1-\cos \vartheta) / 2 .
\end{aligned}
$$

The above sketched model of solar radiation does not violate the formal linearity of (13). Up to now, we have relied on the small changes of $\kappa(),. \lambda(),. \mathcal{F}($.$) and \mathcal{G}($. in (7), with the acceptable values taken from the previous time step in practical calculations, following (13), with some potential improvements a posteriori. Nevertheless, the strongly nonlinear heat radiation by (9) should be discussed and handled separately. In more details: $f^{0}$ and $f^{1}$ in (13) can be written as $\widetilde{f}^{0}+S\left(u^{0}\right)^{4}$ and $\widetilde{f}^{1}+S\left(u^{1}\right)^{4}$ where $(.)^{4}$ means the $4^{\text {th }}$ power of all components of (.) (later also $(.)^{3}$ the $3^{\text {rd }}$ power), $S$ is a sparse diagonal matrix and $\widetilde{f}^{0}$ and $\widetilde{f}^{1}$ are independent of $u^{0}$ and $u^{1}$. The principal difficulty is the poor knowledge of $u^{1}$ a priori (unlike $u^{0}$ ); only some initial estimate $u_{*}^{1}$ is available, e.g. $u_{*}^{1} \approx u^{0}$. For the brevity of notation, let $\widetilde{u}^{1}$ be the result (instead of $u^{1}$ ) obtained from (13) with the correct $f^{0}$, but with $f^{1}$ replaced by $\widetilde{f}^{1}$. Consequently we have

$$
u^{1}=\widetilde{u}^{1}+P S\left(u^{1}\right)^{4} .
$$

where $P=V \Lambda^{-} 1 V^{T}-V(I-\exp (-\Lambda h)) \Lambda^{-2} V^{T} / h$.

The exact Newton method, as derived in [8], pp. 101, 144, applied to (21), gives

$$
\left(I-4 P S\left(u_{*}^{1}\right)^{3}\right)\left(u^{1}-u_{*}^{1}\right)=\widetilde{u}^{1}-u_{*}^{1}+P S\left(u_{*}^{1}\right)^{4},
$$

which can be repeated (with $u_{*}^{1}$ replaced by $u^{1}$ ) in the iteration procedure (if needed, to reach the required accuracy). However, in most application cases the additional term $P S u^{1}$ is not dominating on the right side of $(21)$, consequently the first estimate $u_{*}^{1}$ on the left side of $(22)$ can be taken as fixed, which slightly disturbs the Newton iteration process, but improves the algorithmic efficiency. 


\section{Heating control}

Assuming (for simplicity) the constant temperature in rooms in each time, the conservation of (at least) prescribed temperature level $\bar{u}^{s}(s \in\{1,2, \ldots\})$, e. g. of selected elements of $u$ from (13) corresponding (using the transform (14)) just to rooms with some heating equipments, is required. However, the heating power of these equipments $\mathcal{Q}^{s}$, valid for $(s-1) h<t \leq s h$, is limited, i. e. $0 \leq \mathcal{Q}^{s} \leq \mathcal{Q}^{\max }$ for some upper bounds $\mathcal{Q}^{\max }$ for particular rooms (which is a vector of much lesser length than $n$ in practice). Such upper bounds are design parameters of a building, due to the volume, location and assumed exploitation of rooms.

Consequently we have to distinguish between 4 following artificial heating regimes: 1) no heating during the summer season, 2) inactive heating for sufficient temperature outside the summer season, i. e. $u^{s} \geq \bar{u}^{s}$, 3) normal heating: setting of $\mathcal{Q}^{s}$ is needed, 4) insufficient heating for $\mathcal{Q}^{s} \geq \mathcal{Q}^{s}$ max by 3) when the reduction of $\mathcal{Q}^{s}$ to $\mathcal{Q}^{\max }$ is necessary. Clearly, our principal aim is to evaluate $\mathcal{Q}^{s}$ for the regime 3 ); the modifications for all other regimes are obvious.

Let us compose $W$ from the lines of the matrix $V(I-\exp (-\Lambda h)) \Lambda^{-1} V^{T}$ corresponding to the temperature in rooms with artificial heating, and take $\mathcal{Q}$ as certain additional $\dot{g}$ from artificial heating in (13). Using the least squares technique, we are able to evaluate $\mathcal{Q}^{s}$ from the rather simple formula

$$
W^{T} W \mathcal{Q}^{s}=W^{T}\left(\widehat{u}^{s}-\bar{u}\right)
$$

where $\widehat{u}^{s}$ corresponds to the hypothetical regime 1), active for $(s-1) h<t \leq s h$ (thus $\widehat{u}^{s}$ can be evaluated by (17) directly), and $\bar{u}$ is related (using (14 again) to some prescribed temperature level $\bar{w}$, time-independent here for simplicity (but allowed to have different values in particular rooms, due to the "thermal stability" requirements in modern technical standards, unlike the classical "passive house" evaluation by [9]). We can see that the system of linear algebraic equations (23) is small, thus its solution should be inexpensive; moreover no further inequalities occur in the computational algorithm.

In general, the Newton iterations by (21), including its above discussed modification, disturb the direct evaluation of $\mathcal{Q}^{1}, \mathcal{Q}^{2}, \ldots$ from (23). Fortunately, no additional iterative tricks are needed in most application cases; the corresponding modifications of $\mathcal{Q}^{1}, \mathcal{Q}^{2}, \ldots$ due to $u^{1}, u^{2}, \ldots$ including the heat radiation can be incorporated into the inexact Newton process, as sketched in the preceding section.

\section{Illustrative example}

Our illustrative example presents the thermal analysis of a simple model building, considered as one great room, supplied by building enclosure with particular constructive and insulation layers, located near Brno (Czech Republic); the more complex example of a real building structure will be referenced in Conclusion. All calculations are based on the one-dimensional model simplification, coming from the 


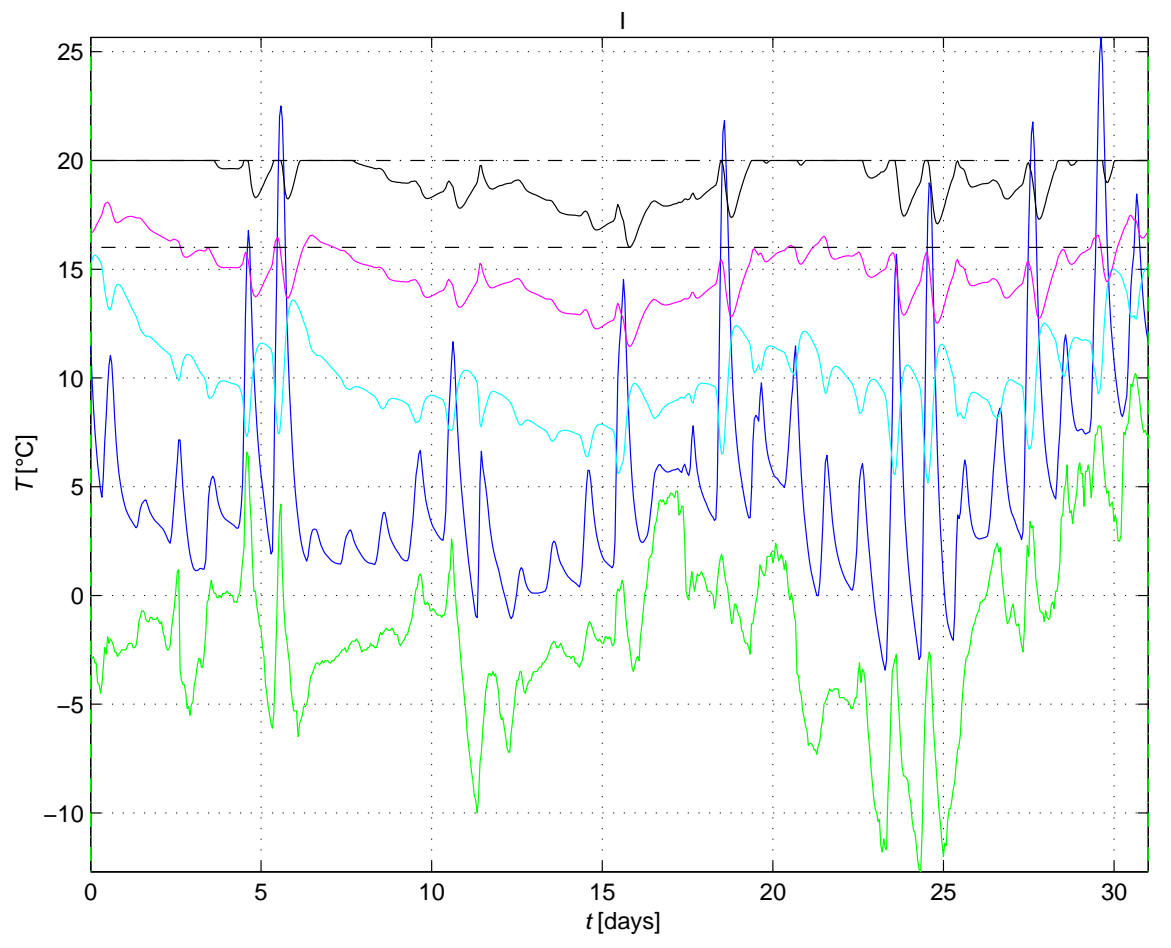

Figure 1: Temperature in a room and structures in January of the $1^{\text {st }}$ year.

linearized heat conduction (no air flow is allowed) and on the application of the finite element method with linear splines as basis functions, with the original software implementation inside the MATLAB environment. Thus it is useful to compare our model with the approach of [22], based on the analogy with the analysis of electrical RC-circuits; a relevant model circuit is described in [15], p.447, and [22], p. 156, in all details. The "lumped masses" by [22] generate diagonal matrices $M$ (in our notation); here we come to (slightly more general) sparse matrices $M$, with technical details explained in [13], p. 62.

The following meteorological data in the time steps $h=1$ hour, averaged to the periodical form of the so-called reference year, from the international airport Brno-Turany are available: i) the environmental temperature, ii) the total solar radiation intensity, iii) the diffusive component of ii). Such data are sufficient to perform all above sketched evaluations, including the annual energy consumption, crucial for the thermal design of a building. The heating is insufficient, with the aim of proper testing of switching between regimes 1), 2), 3), 4). No summer shading (or air conditioning) is installed, to force the unwanted summer increase of temperature in rooms. Three figures document the above sketched direct calculation procedure, starting from the hypothetical inside temperature $u^{0}=20^{\circ} \mathrm{C}$ everywhere. The same minimum temperature level is assumed to be satisfied during the whole year. Visible assertion of the thermal radiation due to the Stefan-Boltzmann law is limited to the slight temperature decrease in some nights. 


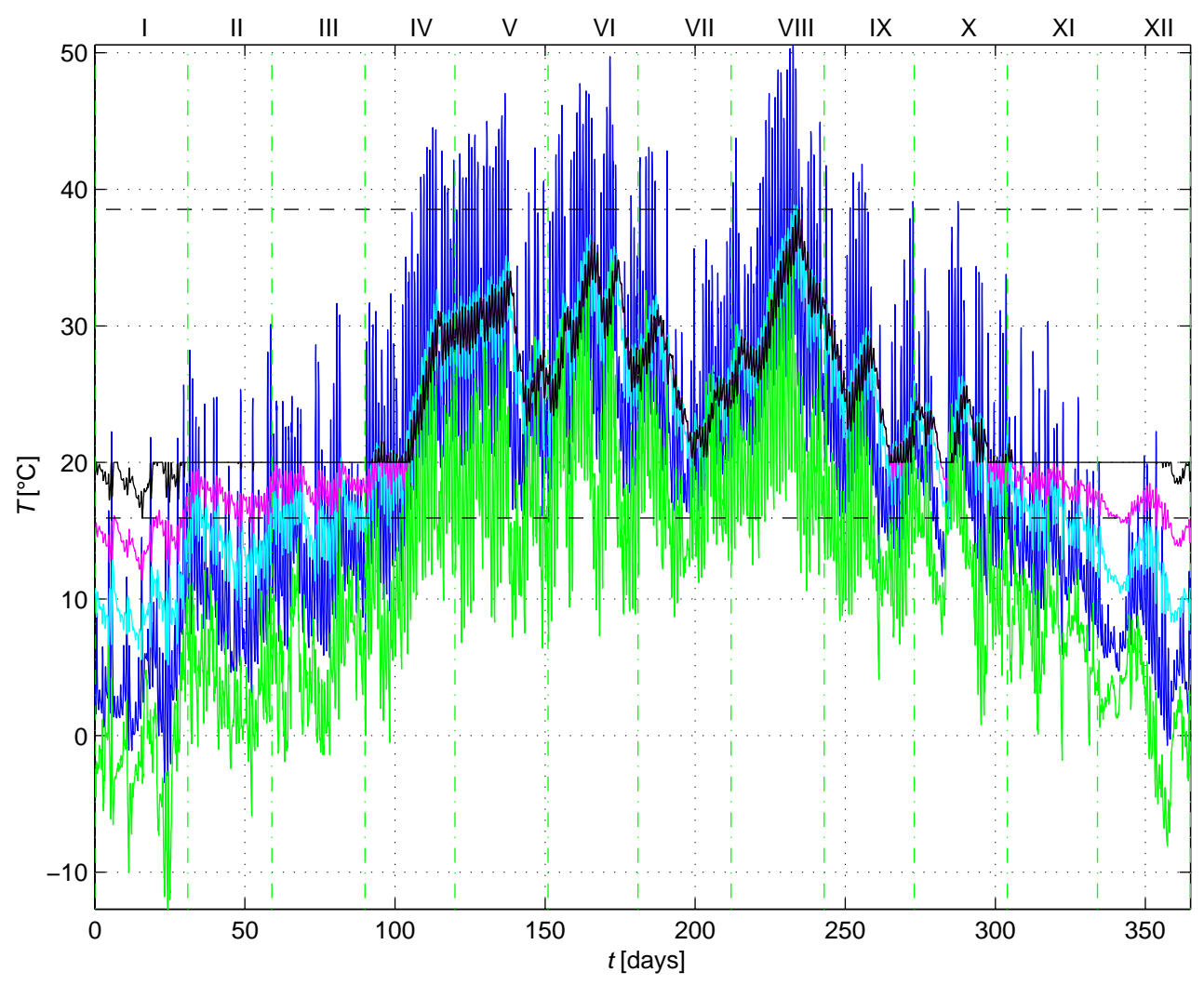

Figure 2: Temperature in a room and structures during the $3^{\text {rd }}$ year.

Figure 1 shows the development of temperature in selected locations of constructive and insulation layers of walls, floors and roof (here just in selected layer centers and interfaces) in the first month (January) of the first calculation year. Namely the lower oscillating curve represents the environmental temperature, the curve rather close to the required $u^{0}=20^{\circ} \mathrm{C}$. The non-negligible decrease of indoor temperature, under $u^{0}=20^{\circ} \mathrm{C}$ corresponds to the strange design of a building for software testing purposes, as introduced above.

The climatic input data are available just for one reference year in practice, thus for simulations considering more years they have to be applied as periodic ones, with the period of 1 year. Apart from the artificial initial condition, one can expect the nearly periodic development of temperature after a small number of years. Figure 2 presents the same computational results as Figure 1, but for the (less synoptic) development of temperature in the third year (the results for the fourth year are nearly the same). The summer overheating of rooms, in some cases even over the prescribed environmental temperature (the maximum inside temperature exceeding $38^{\circ} \mathrm{C}$ occurs in August, which corresponds to the temperature on the outer building surface, oscillating up to $1^{\circ} \mathrm{C}$ ) is caused by the effect of solar radiation, whereas the same radiation in winter, in the direction close to the normal one to most walls and windows, helps to reduce the effect of the insufficient heating. Such idea comes 


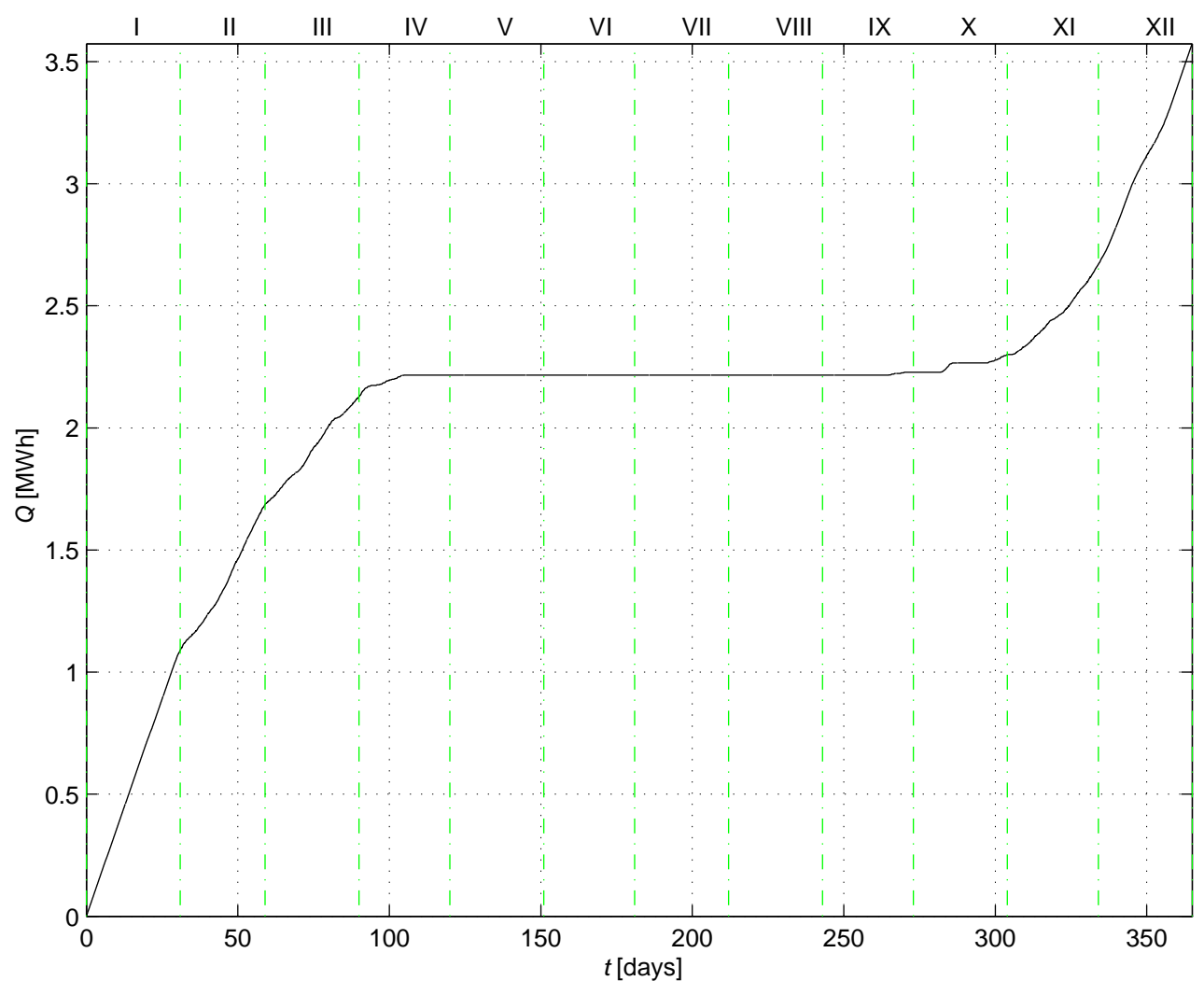

Figure 3: Total consumption of energy for artificial heating during the $3^{\text {rd }}$ year.

even from the ancient literature (Aischylos, Socrates); however, the implementation of some active cooling system (air-conditioning) or at least a passive one (shading) should be expected in modern buildings. More extensive comparative calculations with 34 relevant figures and with the proper discussion of above sketched physical processes are presented in [13], p. 68, including certain identification and optimization considerations.

Finally Figure 3 shows the total consumption of energy, corresponding to Figure 2. Its seemingly regularized increase, with nearly invisible day quasi-cycles, reflects the accumulation ability of applied building materials and structures, as well as the reasonable control of artificial heating. The similar figure corresponding to Figure 1 degenerates just to a linearly increasing function (because of winter climatic conditions together with the low heating power).

\section{Conclusion}

We have demonstrated how the Fourier analysis can contribute to the development of computational algorithms of thermal transfer and related phenomena, including the simplified system analysis of thermal performance of buildings, namely the simulation of energy consumption of building structures, respecting the significant effect of solar radiation and the proper control of heating equipments. The 
corresponding software in MATLAB is still in development; some more references to preliminary results can be found in [12]. The application of this software to various classes of real buildings and the development of further optimization tools belong to the research priorities for the near future.

The robust and inexpensive numerical solver in MATLAB, working with linear algebra, vector operations and spectral decompositions, as sketched under (13), seems to be promising solution for the support of optimization of buildings, both residential and industrial ones, as for the cooling and freezing plants, for the advanced buildings with green roofs and walls (for their practical realization in Vienna see [7]), etc. For example, the evaluation of temperature development and the heating power requirements for the low-energy house in Ostrov u Macochy $(35 \mathrm{~km}$ northern from Brno, some heuristic corrections of climatic data from Brno are needed), with 4 rooms and 26 planar interfaces with the carefully described inner structure, shows the good coincidence between the simulation results and the recorded energy consumption (time series compound from 5 years); for much more details see [13], p. 94. The time step can be still $h=1$ hour, whereas the requirement of stability of the explicit Euler method (in the linearized case) would force $h<161,63 \mathrm{~s}$.

The formal derivation of all convergence properties of implemented algorithms, regardless from their validation from engineering practice, is not trivial, just in the case of iterations caused by strong nonlinearities and optimization steps; the proper analysis are assumed to be prepared for (at least one) another, more extensive paper. The same is true for optimization problems, namely for the application of the Nelder Mead method, using selected functions of the optimization toolbox from MATLAB, to the support of design of building objects by [13], p.89, working with the above mentioned reference object again.

\section{Acknowledgements}

This research has been supported by the project of specific university research of Brno University of Technology, No. FAST-S-16-3385, in cooperation with the project LO1408 AdMaS UP (Advanced Materials, Structures and Technologies, Ministry of Education, Youth and Sports of the Czech Republic, National Sustainability Programme I).

\section{References}

[1] Barták, J., Herrmann, L., Lovicar, V., and Vejvoda, O.: Partial differential equations of evolution. Ellis Horwood, 1991.

[2] Baker, A. and Graves-Morris, D.: Padé approximants. Cambridge University Press, 2010.

[3] Bermúdez de Castro, A.: Continuum termodynamics. Birkhäuser, 2005. 
[4] Bochev, P.B. and Gunzburger, M.D.: Least-square finite element methods. Springer, 2009.

[5] Brenner, S. C. and Ridgway Scott, L.: The mathematical theory of finite element methods. Springer, 2002.

[6] Brigola, R.: Fourier-analysis und distributionen. Co-Verlag, 2012.

[7] Burdajewicz, F., Korjenic, A., and Bednar, T.: Bewertung und Optimierung von dynamischen Dämmsystemen unter Berücksichtigung des Wiener Klimas. Bauphysik 33 (2011), 49-58.

[8] Drábek, P. and Milota, J.: Methods of nonlinear analysis - application to differential equations. Springer, 2013.

[9] Feist, W.: Gestaltungsgrundlagen Passivhäuser. Das Beispiel, Darmstadt, 1999.

[10] Ferziger, J.H. and Peric, M.: Computational methods for fluid dynamics. Springer, 2002.

[11] Gliah, O., Kruczek T., and Thibault, J.: The effective sky temperature: An enigmatic concept. Heat and Mass Transfer 47 (2011), 1171-1180.

[12] Jarošová, P. and Vala, J.: New approaches to the thermal design of energy saving buildings. Advanced Materials Research 1126 (2015), 174-180.

[13] Jarošová, P.: Optimization of selected parameters for the design of objects with low energy demand. (In Czech.) Dissertation thesis, Brno University of Technology, submitted 2017.

[14] Kala, Z.: Sensitivity and reliability analysis of lateral-torsion buckling resistance of steel beams. Archives of Civil and Mechanical Engineering 15 (2015), 10981107.

[15] Kämpf J.H. and Robinson, D.: A simplified thermal model to support analysis of urban resource flows. Energy and Buildings 39 (2007), 445-453.

[16] Nayak, J. K. and Sukhatme, S. P.: Principles of thermal collection and storage. Tata McGraw Hill, 2008.

[17] Pacheco-Vega, A.: Soft computing applications in thermal energy systems. Springer, 2011.

[18] Roubíček, T.: Nonlinear partial differential equations with applications. Birkhäuser, 2005. 
[19] Škrjanc, I., Župančič, B., Furlan, B., and Kainer, A.: Theoretical and experimental fuzzy modelling of building thermal dynamic response. Building and Environment 36 (2001), 1023-1038.

[20] Štastník, S., and Vala, J.: On the thermal stability in dwelling structures. Building Research Journal 52 (2004), 31-55.

[21] Sukhatme, S. P.: Solar energy. Tata McGraw Hill, 1996.

[22] Underwood J. C.: An improved lumped parameter method for building thermal modelling. Energy and Buildings 79 (2014), 191-201.

[23] Vala, J.: Computational approaches to some inverse problems from engineering practice. Programs and Algorithms of Numerical Mathematics 17 in Dolní Maxov (Czech Republic, 2014), Institute of Mathematics CAS, Prague 2015, $215-230$.

[24] White, R.E.: Elements of matrix modeling and computing with MATLAB. Chapmann \& Hall / CRC, 2005.

[25] Directive 2010/31/EU of the European parliament and of the council on the energy performance of buildings. Official Journal of the European Union L 153/13, 2010. 